\title{
LA CONFESIONALIDAD DEL ESTADO COSTARRICENSE: UN PROCESO EN CONSTANTE RENOVACIÓN
}

\section{THE CONFESSIONALITY OF THE COSTA RICAN STATE: A PROCESS IN CONSTANT RENOVATION}

\author{
Adriana Maroto Vargas ${ }^{1}$ \\ adriana.maroto_v@ucr.ac.cr \\ adriana.maroto.vargas@gmail.com
}

\begin{abstract}
Resumen
En este artículo se abordan tres grandes ejes. Primero, una discusión teórica sobre los conceptos de laicidad y secularización desde el marco de la sociología de la religión. Segundo, el papel particular que ha jugado la jerarquía católica en la configuración del Estado en América Latina. Por último, se presenta un balance general sobre la relación histórica entre el Estado y la jerarquía católica en Costa Rica. Se concluye que en Costa Rica, la jerarquía católica más que como un grupo de presión debe entenderse como un actor de poder e influencia significativa en el desarrollo de la cultura y del Estado. De esta manera, la confesionalidad del Estado, más que un resabio histórico debe entenderse como un proceso en constante renovación.

Palabras claves: laicidad, secularización, Estado confesional, Costa Rica, neoliberalismo.
\end{abstract}

\begin{abstract}
This article addresses three main subjects. First it presents a theoretical discussion of the concepts of secularism and secularization from the focus sociology of religion. Second, the particular role played by the Catholic hierarchy in the configuration of the state in Latin America. Finally, it presents a general balance on the historical relationship between the state and the catholic hierarchy in Costa Rica. It concludes that in Costa Rica, the catholic hierarchy rather than as a pressure group should be understood as an actor of power and significant influence on the development of culture and the state. Thus the confessional state rather than a historical relic must be understood as a process in constant renovation.
\end{abstract}

Key words: secularism, secularization, confessional state, Costa Rica, neoliberalism.

1 Docente e investigadora en la Escuela de Psicología, Universidad de Costa Rica, Sede Rodrigo Facio 


\section{Introducción}

Hechos recientes como las negociaciones para la firma de un Concordato entre Costa Rica y la Santa Sede, las discusiones en torno a la reforma del artículo constitucional que establece la religión católica en Costa Rica como la oficial del Estado, la declaratoria de Laura Chinchilla Miranda como hija predilecta de la Virgen María, la designación de la Virgen de los Ángeles como comandante del Servicio de Vigilancia Área de la República de Costa Rica y la consagración realizada por las personas que ejercen la Presidencia de los tres poderes de la República en la ceremonia de la Virgen de los Ángeles en el año 2013, hacen que la discusión sobre la laicidad en Costa Rica mantenga una gran vigencia y relevancia.

Desde su origen como República independiente Costa Rica ha sido un Estado confesional, sin embargo, se han suscitado cambios históricos respecto a la relación que existe entre representantes del Estado y la jerarquía católica. En el país, se ha investigado sobre la relación de estas dos instituciones desde la época colonial, evidenciando la fuerte influencia mutua que tuvieron para establecerse en los inicios de la República.

Es a través de este proceso histórico que se ha "naturalizado" la participación de la jerarquía de la iglesia católica en el espacio público y especialmente en eventos políticos muy particulares como actos oficiales del gobierno y la toma de posesión de la Presidencia de la República, momentos en que el montaje de ese escenario simbólico se convierte en una importante herramienta de legitimación del poder político y del poder eclesiástico.

En este artículo se presentan reflexiones teóricas sobre los conceptos de laicidad y secularización, el papel particular que ha jugado la jerarquía católica en la configuración del Estado en América Latina, así como un balance general sobre la relación histórica entre el Estado y la jerarquía católica en Costa Rica. Las mismas son parte de la tesis de la Maestría Académica en Sociología titulada Los procesos de construcción de la legitimidad en las relaciones Estado - iglesia católica en Costa Rica, 2007-2010, presentada a finales del 2012.

\section{Los debates teóricos en torno a la secularización y la laicidad}

Si bien el concepto de secularización tiene raíces históricas que datan de hace algunos siglos, es en el siglo XX que el secularismo emergió como una categoría científica, en este caso en el campo de la sociología de la religión a partir de la obra de Max Weber.

Tschannen (2004) argumenta que definir la secularización es muy difícil, debido a que se presenta como un concepto de alcance universal, en tanto busca explicar una realidad muy compleja y bastante amplia. Esta podría ser una de las razones por las cuales las discusiones teóricas sobre este tema han sido a su vez amplias y complejas.

Durante los años sesentas y setentas del siglo XX hubo un importante auge de las teorías de la secularización a través de múltiples enfoques y propuestas de autores como Thomas Luckmann, Peter Berger, Bryan Wilson, David Martin, Richard Fenn, Talcott Parsons, Robert Bellah, Rodney Stark y Niklas Luhmann, entre otros.

Estruch (1996) plantea una síntesis de cómo se pueden agrupar estas teorías de la secularización. Otros autores pueden plantear modelos diferentes, lo importante es -para efectos académicos- evidenciar los diversos alcances o énfasis que han tenido las propuestas teóricas elaboradas.

Un primer grupo son aquellas que consideran que la secularización expresa la creciente decadencia de la religión y apunta a su próxima desaparición. Esta propuesta parte de una confusión entre religión e iglesia, con lo cual se llega a la conclusión de que una crisis de la institución eclesiástica tendría como causa directa una crisis definitiva de la religión (Estruch, 1996).

El segundo grupo está integrado por las propuestas teóricas en que la secularización refleja el proceso de progresiva "mundanización" de lo religioso. Se expresa a través de los esfuerzos de modernización y adaptación de las instituciones religiosas a los cambios sociales (Estruch, 1996; Mallimaci, 2004).

Un tercer grupo son las teorías en las que la secularización es un proceso en que la sociedad adquiere autonomía e independencia frente a lo religioso. Mediante un proceso de diferenciación gradual, se sustrae el papel de la religión en la 
vida pública y la coloca en el ámbito de la vida privada (Estruch, 1996).

Finalmente, las teorías que plantean la secularización como una desacralización del mundo. El mundo en su complejidad queda desvinculado del carácter sagrado, de tal manera que este adquiere su significados a través de explicaciones de tipo racional-causal. Esta propuesta fue planteada por autores clásicos de la sociología como Comte, Marx y Weber; sin embargo, este último advirtió que este desencantamiento sería un empobrecimiento, ya que ni la ciencia ni la razón podrían dar cuenta del sentido último de las cosas (Estruch, 1996).

Con diferentes énfasis, el núcleo común es que las teorías que abogaban por la secularización pronosticaron el fin de la era en que la religión y los símbolos sagrados otorgaban sentido a la vida de las personas y las sociedades, cediendo este lugar a la razón y la ciencia, con lo cual, se daría un cambio fundamental en los símbolos y la cultura (Donoso, 2008; Martín Huete, 2007).

Es así como la secularización se planteó como norma de validez universal y el ideal a alcanzar mediante la modernidad. Era una noción lineal y continua de este ideal de progreso. Sin embargo en el análisis histórico queda claro que fue solo un fenómeno occidental, principalmente restringido a las sociedades europeas (Estruch, 1996).

Por lo tanto, estas teorías deben entenderse en el contexto histórico en el cual fueron propuestas, ya que esto permite entender las particularidades de este proceso en América Latina en general y Costa Rica en particular. Donoso (2008) plantea la secularización como un proceso que ha estado diferenciado geográficamente, si bien surgió en Europa, ha seguido desarrollos diversos en el resto del mundo.

Martín Huete (2007) plantea que de acuerdo con Berger, los procesos de secularización tienen tres localizaciones geográficas con procesos y resultados diferentes. A saber,

1. Europa (principalmente central y occidental): es donde mejor se ha desarrollado la teoría de secularización principalmente a nivel institucional. Este modelo pierde su sentido si se trata de exportar a otros lugares.
2. Estados Unidos: convive una sociedad moderna con una sociedad muy religiosa, lo cual hace más complejo el desarrollo de una teoría de la secularización. Históricamente, la religión ha tenido una relación estrecha con el sistema de clase (pertenecer a la iglesia protestante es un signo de estatus de la clase media) y ha servido como agente de movilidad social. El proceso se ha complejizado en tanto se ha pluralizado la sociedad estadounidense (Martín Huete, 2007).

3. Resto del mundo (incluyendo América Latina): a pesar que desde mediados del siglo XVIII se extiende la idea de la secularización de otras regiones del mundo, existe un fuerte fervor religioso en dos sentidos. Por un lado, una persistencia de la religión tradicional y por otro el resurgimiento del fundamentalismo religioso, todo lo cual afecta la teoría de la secularización (Martín Huete, 2007).

En la segunda mitad del siglo XX, y con mayor énfasis a partir de la década de los setentas, los autores empezaron a señalar que hubo un retorno de lo religioso. En otras palabras, la religión volvió a tomar un papel central como mecanismo que otorga sentido a la existencia de las personas (Martín Huete, 2007).

Tschannen (2004) se refiere a esto como un efecto boomerang, ya que se generaron movimientos de contrasecularización, cuya fuerza es proporcional a la de los movimientos precedentes. Esto es bastante visible en la región latinoamericana con la fuerte presencia de los fundamentalismos religiosos en temas relativos a los derechos sexuales y los derechos reproductivos por ejemplo.

Por otro lado, para abordar el tema de la secularización debe abordarse la laicidad. Si bien son conceptos y procesos que están muy relacionados, debe entenderse su diferencia conceptual. La laicidad, en su sentido más estricto, designa la separación entre el Estado y las iglesias, en el caso de América Latina particularmente la iglesia católica que es la que ha ostentado el poder desde la época colonial (Vallarino, 2005).

La laicidad es uno de los componentes de la secularización, pero a diferencia de esta, no abarca la amplitud de la relación entre política y 
religión sino la especificidad entre la institución política (el Estado) y las instituciones religiosas (las iglesias) (Tschannen, 2004).

Es fundamental entender que la laicidad es un tema propio de la sociología de la religión: debe discutirse como un fenómeno político y no como un problema religioso. Es decir, desde esta óptica se entiende que el Estado es el responsable de establecer los parámetros para que la laicidad sea viable. Vallarino (2005) afirma que la iglesia no se puede oponer a la laicidad en la medida en que el Estado sea capaz de definirla. Dicho en otras palabras, la ausencia del carácter laico es un signo de incapacidad o insuficiencia del Estado.

La laicidad se puede definir en torno a dos ejes complementarios: laicidad-separación y laicidad-neutralidad. En tanto existe esta separación formal entre el Estado y la iglesia, se entiende como un Estado laico. Esto, teóricamente, significa que el Estado es independiente de toda religión y no admite ser influido por ella en la toma de sus decisiones. Para la iglesia, implica que es libre y autónoma para actuar en el ámbito de la sociedad civil, para todas las personas quienes existe garantía del ejercicio de la libertad religiosa y la libertad de conciencia (Vallarino, 2005).

La noción de neutralidad implica no solamente que el Estado no profesa o sostiene ninguna confesión, sino que además, no se pronuncia ni interviene en materia religiosa, es decir, el Estado admite las manifestaciones religiosas existentes y no toma disposiciones que afecten su organización e independencia, siempre y cuando estas no afecten normas básicas de convivencia (Vallarino, 2005).

Para que esto sea factible, el Estado tendrá que crear un marco jurídico donde reconozca y garantice la libertad de conciencia y la libertad religiosa que, como principios constitucionales, existen en la mayoría de los países latinoamericanos.

\section{Influencia de la jerarquía católica en la configuración del Estado en América Latina}

Según Bourdieu (1988), “el Estado es el resultado de un proceso de concentración de los diferentes tipos de capital (...) que convierte al
Estado en poseedor de una especie de metacapital, otorgando poder sobre las demás clases de capital y sobre sus poseedores" (p. 99). En este proceso se elaboran los campos de poder, entendido por el autor como el espacio en el cual se libra la lucha por el poder sobre el capital que se ha de imponer y reproducir. En este sentido y respecto al tema de interés en este artículo, es de gran importancia la participación activa de los agentes religiosos en la vida pública, a fin de que el capital religioso ocupe un lugar privilegiado.

En la complejidad teórica sobre el tema del Estado, se retoma este autor, debido a que interesa destacar el papel del Estado en la unificación de los códigos culturales, con lo cual se moldean las estructuras mentales que permiten la consolidación de la identidad nacional (Bourdieu, 1988).

En América Latina y particularmente en Costa Rica, la iglesia católica ha jugado un papel muy importante en la configuración de la identidad nacional a través de una alianza con las y los representantes del Estado que ha tenido como consecuencia la imposición de una moral con fuertes cimientos religiosos.

Kaplan (1984) aduce que las raíces de la configuración de los Estados latinoamericanos se encuentran en el período colonial. Durante esta época, el proceso de evangelización e imposición de la religión católica fue muy importante para consolidar la dominación. Como consecuencia, el proceso de configuración política de los nuevos Estados estuvo muy ligado a la consolidación de la iglesia católica en la región.

Siguiendo la huella de estas raíces, una etapa muy importante para la formación del Estado se ubica en el siglo XIX. Las principales tareas para lograrlo fueron el establecimiento de "alianzas, la construcción del orden político e institucional, la definición de las funciones estatales de legalidad, coacción social y la constitución de la clase dominante", entre la cual se encontraba la jerarquía católica (Kaplan, 1984, p. 28).

En América Latina se impuso el modelo de Estado proveniente de Europa y Estados Unidos caracterizado por ser un Estado centralizado, basado -formalmente- en la soberanía popular y la democracia representativa. Sin embargo, en la región persistían con gran fuerza las tradiciones locales (incluyendo una fuerte religiosidad), 
profundas divisiones raciales, étnicas y sociales, y finalmente, una institucionalidad que no tenía la capacidad ni la legitimidad requerida para hacer efectivo este modelo en la región (Kaplan, 1984; Pérez, 2007).

Este proceso generó una fuerte tensión entre estas dos realidades: un Estado moderno diseñado bajo los ideales de libertad, justicia y democracia, versus la otra, caracterizada por el autoritarismo, la injusticia y la desigualdad. Según Pérez (2007), esta última "es propia de una cultura religiosa y política premoderna que domina la región aún en el siglo XXI" (p. 79).

Pérez (2007) considera que es usual que los estudios relacionados al Estado y al desarrollo político latinoamericano hayan ignorado esta contradicción entre la modernidad formal del Estado y la premodernidad cultural predominante en América Latina. En algunos casos, se ha estudiado la influencia que tienen las organizaciones religiosas, incluyendo la iglesia, cuando actúan como grupos de presión; sin embargo, se ha desestimado el poder y la influencia significativa que ha tenido la religión en el desarrollo del Estado y la cultura política de la región.

En nuestro medio es bastante frecuente que las prácticas políticas no estén secularizadas: los símbolos religiosos tienen un gran poder y el orden moral sigue siendo en general dictado por imperativos religiosos. Aunque es evidente en formas muy diversas, es muy claro en cuanto a los símbolos marianos tal y como es muy evidente en el país en torno a la Virgen de los Ángeles (Bastian, 2004).

A diferencia de otras regiones del mundo, por ejemplo Europa, en América Latina la noción de dios que existe establece una visión providencialista de la historia, es decir, se considera que la historia de las personas y las sociedades, en todos los aspectos, es gobernada por dios de acuerdo a sus planes. Dicho en otras palabras, la realidad social está determinada por fuerzas que escapan a la acción social. En Costa Rica, por ejemplo, según una encuesta de la empresa Unimer realizada para el periódico La Nación en octubre del 2011, un $64 \%$ de las personas considera que su futuro depende de dios (Araya, 2011).

Tener en cuenta este aspecto para el análisis del desarrollo político en la región es muy importante porque esta actitud genera -en términos generales- conductas pasivas ante la desigualdad social, la corrupción y otros temas políticos (Pérez, 2007).

Durante las últimas décadas, la influencia del neoliberalismo ha provocado una reorganización del Estado en América Latina de acuerdo a la presión del mercado global y los organismos financieros internacionales. Sin embargo, afirma Pérez (2007), el neoliberalismo no debe entenderse únicamente como un proceso económico sino también cultural y definidor de una ética.

Para este autor, el providencialismo característico de la iglesia católica, se ha alineado con la cultura neoliberal, "reforzando los aspectos más dañinos de la racionalidad instrumental en base a la cual se organiza y expande el mercado" (Pérez, 2007, p. 92). Es así como el modelo neoliberal, encuentra una lógica social que cree que el mundo es un espacio colonizado por fuerzas externas que lo definen todo. Por lo tanto, la idea de un orden abstracto, complejo, espontáneo, autogenerado y autorregulado encuentra un fuerte asidero en la cultura.

Es en este contexto histórico de la configuración del Estado en América latina que debe entenderse el proceso de laicidad que se ha generado en la región, con características y resultados muy diferentes a lo acaecido en Europa. En términos muy generales, retomando a Mallimaci (2004) el proceso de laicidad en América Latina se puede entender en tres momentos.

El primero de ellos, ubicado de 1880 a 1930, período en que los países construyeron sus Estados con la influencia de la ideología liberal, la cual propició enfrentamientos con la jerarquía católica. No obstante, los grupos en el poder tenían claro la importancia de la vinculación con los religiosos para la consolidación del poder político. Afirma el autor, que no existía esencialmente una actitud antirreligiosa, sino que se perseguía la separación entre el Estado y la iglesia con el objetivo de debilitar el poder clerical para construir sociedades más pluralistas.

El segundo período comprende de 1930 a 1980, etapa en que el modelo a seguir en la región fue el Estado de bienestar. En este período las y los representes del Estado identificaron la 
necesidad de establecer alianzas políticas que fortalecieran su legitimidad en el poder.

Para este período existía una mayor pluralidad religiosa en la región debida al surgimiento y crecimiento de otros grupos religiosos. No obstante, el catolicismo y su jerarquía sigue siendo el actor hegemónico, el cual aparece como un dador de identidad nacional y cultural que legitima la dominación. Por lo tanto, se busca la recuperación del espacio de la jerarquía católica en la vida política, para lo cual no fue necesario realizar modificaciones jurídicas a la separación entre ambas instituciones, establecida en la mayoría de los países durante finales del siglo anterior (Mallimaci 34, Bastian, 2004).

Actualmente a nivel constitucional, Chile, Colombia, Ecuador, El Salvador, Guatemala, Honduras, México y Venezuela se "limitan" a establecer la libertad de religión y culto. Mientras que Cuba, Bolivia y Puerto Rico estipulan que existe separación o independencia entre las instituciones religiosas y el Estado; Nicaragua y Paraguay señalan que no existe una religión oficial y Uruguay establece que el Estado no sostiene ninguna religión. Estos últimos seis países mencionados son los que podría considerarse, normativamente hablando, que establecen la laicidad de su Estado.

En el caso de República Dominicana, Perú y Brasil, constitucionalmente no se otorgan privilegios a la iglesia, sin embargo, han suscrito concordatos (1954, 1980 y 2009 respectivamente) que sí lo hacen. Por su parte, Argentina y Panamá otorgan constitucionalmente algunos privilegios a la iglesia católica, como por ejemplo el reconocimiento de que es la religión que practica la mayoría de la población o el financiamiento directo. Costa Rica es el único país de la región que constitucionalmente reconoce una religión como oficial del Estado.

No obstante, el estatus jurídico no brinda una garantía sobre la laicidad real de cada Estado. Tschannen (2004) afirma que existe una debilidad jurídica de la laicidad en América Latina, ya que al margen de lo establecido en la norma jurídica, los grupos religiosos continúan siendo interlocutores en el debate político y el Estado se apoya en una legitimidad brindada por el aparato religioso para incrementar su influencia social.
Cobre así gran relevancia el concepto de laicidad positiva propuesto por Orozco (2011), la cual caracteriza como un disfraz de un Estado confesional bajo la bandera de la laicidad, ya que en este caso, el Estado establece ciertas políticas $\mathrm{o}$ actividades de fomento de ciertas actividades y grupos religiosos a quienes se les brinda un trato privilegiado y por lo tanto, son claramente discordantes con el concepto de laicidad enunciado anteriormente.

Finalmente, el tercer período para el análisis de la laicidad en América Latina inicia en 1980 a partir de la crisis del Estado benefactor. El avance de la modernidad y del capitalismo neoliberal fue considerado por los sectores religiosos conservadores como aquellos factores que facilitaron la expansión del secularismo y con este, la destrucción de la familia y los valores tradicionales (Mallimaci, 2004; Bastian, 2004).

En estos últimos 30 años en América Latina ha habido una tensión en el monopolio de la administración de los bienes de salvación debido a la rápida emergencia y consolidación de otros actores religiosos. No obstante, a pesar de ser una lucha compleja entre varios actores en un mercado de bienes simbólicos abierto, la iglesia católica sigue teniendo un estatus privilegiado en el plano político y Costa Rica no es la excepción (Bastian, 2004).

En el caso particular de Costa Rica, desde la época colonial hasta 1884 hubo lo que Picado (1990) denomina una cristiandad colonial en el cual se dio la consolidación del catolicismo en el país, incluyendo la firma de un Concordato en el año 1852. Según Benavides (2005), en el acuerdo entre el Vaticano y el gobierno costarricense, la mayoría de los aspectos beneficiaban a la iglesia. En 1852 inició un nuevo período que Picado define como la nueva cristiandad liberal, la cual se extendió hasta el año 1892.

En esta última mitad del siglo XIX tuvieron gran relevancia los ideales liberales, los cuales marcaron uno de los momentos más críticos en la relación entre representantes del Estado y la jerarquía católica en el país. Los liberales abogaban por la libertad de culto y en el año 1884 se aprobaron las llamadas leyes anticlericales.

Entre ellas, se derogó unilateralmente el Concordato, se produjo la secularización de los 
cementerios, se instauró la prohibición de realizar celebraciones religiosas en sitios públicos, se aprobó el divorcio y el matrimonio civil, se proclamó el desconocimiento de los votos religiosos, se fortaleció la enseñanza laica y finalmente, se expulsó al obispo Bernardo Augusto Thiel (Benavides, 2005; Campos, 2000; Picado 1990).

En este período, si bien hubo cambios muy importantes, no hubo reforma del artículo constitucional que establecía la confesionalidad del Estado. En períodos posteriores hubo nuevos acercamientos entre ambas instituciones, que según Campos alcanzaron su punto más alto en la década de los años cuarenta del siglo XX.

De 1892 a 1940 se dio un ciclo de convivencia pacífica, en el cual la jerarquía católica recuperó su posición cerca del Estado, existiendo una armonía entre ambas instituciones. La iglesia empezó a tener un lugar importante en la vida política desde la década de los años veinte, sin embargo llegó a su momento de mayor auge en la década de 1940, cuando se inició la transición de los gobiernos liberales al modelo de Estado social de derecho (Benavides, 2005; Campos, 2004; Picado, 1990).

A partir de la segunda mitad del siglo XX, la jerarquía de la iglesia católica estableció una relación simbiótica con el Estado, esto en cuanto ha sido una asociación en que las dos partes se han beneficiado. Esta vinculación ha permitido - según Picado (1990) - que el Estado oriente el accionar de la iglesia, la cual ha guardado "una dependencia con el proyecto desarrollista impulsado por el Partido Liberación Nacional" (p. 43).

Debido a esta vinculación política, la jerarquía de la iglesia católica se ha centrado en la clase media, ya que es una estrategia que por un lado permite neutralizar la lucha de clases, pero principalmente porque es un medio óptimo para el mantenimiento de la moral familiar que ayuda a mantener el status quo (Picado, 1990, p. 79).

El período de 1949 a 1979 se caracterizó por una cristiandad reformista, en la cual el Estado legisló a favor de la iglesia católica y solicitó su apoyo para el mantenimiento del orden social. La década de los cuarentas fue muy importante porque en el gobierno de Rafael Ángel Calderón Guardia, ambas instituciones plantearon que existía una armonía entre el ideal católico de justicia social y la labor gubernamental, lo cual justificó una participación activa del clero en la vida política.

La Asamblea Constituyente de 1949 discutió tres aspectos importantes: la participación política del clero, la confesionalidad del Estado y la educación. En este proceso se habilitó a los clérigos a ser electos como diputados pero se restringió su participación para los cargos de la Presidencia y Vicepresidencia de la República, Ministerios y Magistrados de la Corte Suprema de Justicia. La confesionalidad del Estado, quedó establecida en el artículo 75 de la Constitución Política, adoptando el mismo texto de la Constitución anterior sin mayor discusión. Y, finalmente, se garantizó la participación e influencia de la iglesia en el diseño y supervisión de los programas de enseñanza. Con todo ello, se puede decir que la Constitución de 1949 no introdujo cambios significativos en las relaciones Estado e iglesia católica (Benavides, 2005; Campos, 2000).

El tercer ciclo identificado por Picado se denomina ciclo de la práctica eclesial neoconservadora e inició en 1979, año que fue nombrado arzobispo de San José Román Arrieta Villalobos. El inicio de la década de los ochentas fue un momento político muy importante debido a que el gobierno tenía como gran reto superar la crisis en medio de la cual estaba inmerso el país. La iglesia asumió un papel público frente a los temas sociales y políticos, de tal manera que fue un actor importante en la intermediación de conflictos laborales (Campos, 2000).

En las administraciones liberacionistas de Monge y Arias durante la década de los años ochenta, los Presidentes solicitaron la participación de la jerarquía católica en el proceso de diálogo entre los sectores sociales, estrategia definida por los gobiernos para enfrentar la crisis a la que se enfrentaban. La participación en este proceso fue la contribución de la iglesia que se consideró como más importante, sin embargo, acota Picado (1990) que fue el aval del obispo Román Arrieta al sistema capitalista.

A inicios de la década de los noventas, con el gobierno de Rafael Ángel Calderón Fournier por el Partido Unidad Social Cristiana (19901994) se dio una relación muy tensa entre ambas instituciones, particularmente por la discusión 
pública de temas relativos a los derechos sexuales y los derechos reproductivos (Campos, 2000). No obstante, en este período se aprobó el Reglamento sobre el Otorgamiento y la Revocatoria de la Missio Canónica, con el cual se otorgó a la CECOR el monopolio sobre la educación religiosa en el sistema de educación formal.

En el caso de la administración de José Figueres Olsen (1994-1998) el tono de la tensión bajo un poco, como fue característico de los gobiernos de Liberación Nacional de finales del siglo XX. A pesar de esto, se mantuvieron algunos desacuerdos respecto a la política económica y especialmente por el reconocimiento de la Unión de Hecho en el año 1995 (Campos, 2000).

En la administración de Miguel Ángel Rodríguez del Partido Unidad Social Cristiana (1998-2002) hubo dos momentos de gran tensión entre el gobierno y sectores de la iglesia católica: la discusión del Proyecto de Ley para la modernización y mejoramiento de los servicios públicos de electricidad y telecomunicaciones y de la participación del Estado (conocido como Combo del ICE) y dos programas impulsados por la Primera Dama Lorena Clare que abordaban temas de educación para la sexualidad: Amor Joven y Construyendo Oportunidades.

El mandatario Abel Pacheco de la Espriella del Partido Unidad Social Cristiana, en su gestión (2002-2006) inició la implementación de la política de sexualidad mediante la capacitación de docentes a través del recién creado Departamento de Educación Integral de la Sexualidad Humana del MEP. Debido a situaciones internas de la iglesia católica, se presume que la Conferencia Episcopal no tuvo una participación activa en este proceso. No obstante, en el 2003 se firmó un convenio entre el MEP y la CECOR en donde se otorgaron algunos privilegios como el traslado de recursos financieros y el reconocimiento de la Universidad Católica (Fernández, 2010).

Posteriormente, el gobierno de Oscar Arias Sánchez (2006-2010) del Partido Liberación nacional, se caracterizó por una relación tensa con los obispos a raíz de la discusión del Tratado de Libre Comercio. No obstante, en este período se hizo un proceso de restructuración interna del Ministerio de Educación Pública que concluyó con la desintegración del departamento encargado de la educación para la sexualidad (Fernández, 2010).

Mediante esta muy breve reseña, se puede concluir que en los últimos veinte años, los temas que han generado mayor participación de la jerarquía católica en la vida política del país, ha sido la discusión de políticas, planes, programas o leyes relacionadas con los derechos sexuales y los derechos reproductivos de las personas y la educación religiosa en el sistema público de educación.

Además, se puede apreciar que las relaciones entre representantes del Estado y la jerarquía católica, han estado marcadas por el disenso, el conflicto, los desacuerdos y la tensión. No obstante, existe una negociación constante -la cual no se hace explícita frente a la opinión públicaque permite llegar a acuerdos.

En este punto se concuerda con el planteamiento bergeriano de que la religión es uno de los mecanismos legitimadores de las instituciones sociales más eficientes que existen, especialmente, porque no se trata de un intercambio recíproco incondicional, sino que es un intercambio estratégico que preserva los intereses de las instituciones.

\section{Conclusiones}

A través de las reflexiones anteriormente planteadas, se puede concluir que la relación entre el Estado y la iglesia católica en el país es compleja y que la confesionalidad del Estado, lejos de ser una disposición constitucional que ha perdido vigencia, mantiene una gran actualidad y se renueva de manera constante en un intenso proceso de negociaciones entre el gobierno de cada período y la jerarquía católica.

Por lo tanto, se puede concluir que la jerarquía católica más que como un grupo de presión debe entenderse como un actor de poder e influencia significativa en el desarrollo del Estado y la cultura política en América Latina.

En el caso de Costa Rica, la Conferencia Episcopal ha jugado su papel particular como actor político en la gestión y consolidación del modelo económico vigente en el país. Aún cuando algunos voceros de la jerarquía católica han se han manifestado en contra del modelo neoliberal en momentos particulares como por ejemplo la 
ratificación del Tratado de Libre Comercio con Estados Unidos, la CECOR como ente colegiado ha dejado de ser crítica del mismo y en su lugar se ha mantenido al margen de la discusión con un silencio cómplice.

Donoso afirma que en América Latina la relación entre el Estado y la iglesia católica se ha dado en función de una lógica corporativista. Esta ha permitido mantener un modelo económico que genera grandes ganancias para ciertos sectores sociales a los que pertenecen tanto las y los representantes del Estado como a la jerarquía católica.

$\mathrm{Al}$ abordar el tema del neoliberalismo este no debe entenderse únicamente en su dimensión económica, sino también en tanto definidor de un marco ético y cultural. Surge la pregunta sobre el papel que juega la familia como grupo social en este modelo económico. No cabe la menor duda que la familia, entendida en su acepción más tradicional, es un bastión de gran importancia para el mantenimiento del status quo y la moral universal que interesa a la jerarquía católica pero por otro lado surge la pregunta, jes acaso la familia una base social para mantener el status quo desde un punto de vista económico que interesa a las y los representantes del Estado?

En este artículo no se puede dar respuesta categórica a esta interrogante, sin embargo, no puede obviarse que otros estudios han planteado que las familias han sido el principal soporte de bienestar para las personas en el marco del cambio del Estado benefactor al Estado neoliberal. Por lo tanto, queda planteada la interrogante sobre este tema como una línea de investigación.

\section{Referencias bibliográficas}

Araya, A. (2011, Septiembre 9). 41\% de costarricenses apoya un Estado sin religión oficial. La Nación. Recuperado de http://www. nacion.com

Bastian, J.P. (2004). La recomposición religiosa de América Latina en la modernidad tardía. En J.P. Bastian (coord.), La modernidad religiosa. Europa latina y América Latina en perspectiva comparada. (pp.155-174). México D.F.: Fondo de Cultura Económica.
Berger, P. (2006). El dosel sagrado. Para una teoría sociológica de la religión. 5ta edición. Barcelona: Editorial Kairós.

Bourdieu, P. (1988). Cosas dichas. Barcelona: Editorial Gedisa.

Campos, D. (2000). Relaciones Iglesia-Estado en Costa Rica: un estudio histórico-jurídico. San José: Editorial Guayacán.

Donoso Maluf, F. (2008). El porvenir de una des-ilusión: Hacia un examen pluriaxial de la secularización. Teología y vida. XLIX, 799-835.

Estruch, J. (1996). El mito de la secularización. En R. Díaz Salazar (Ed.), Formas modernas de secularización. (pp.266-280). Madrid: Alianza Universidad.

Kaplan, M. (1984). Estado y sociedad en América Latina. Ciudad de México: Editorial Oasis.

Mallimaci, F. (2004). Catolicismo y liberalismo: las etapas del enfrentamiento por la definición de la modernidad religiosa en América Latina. En J.P. Bastian (coord.), La modernidad religiosa. Europa latina y América Latina en perspectiva comparada. (pp.155-174). México D.F.: Fondo de Cultura Económica.

Martín Huete, F. (2007). El problema de la secularización en el pensamiento de Peter L. Berger: de la secularización a la desecularización. ¿Hacia un cambio de paradigma religioso? (Tesis doctoral no publicada). Departamento de filosofía. Facultad de Filosofía y Letras. Universidad de Granada. Granada: Universidad de Granada.

Orozco Solano, V.E. (2011). Laicidad y Libertad de Religión. San José: ISOLMA.

Pérez Baltodano, A. (2007). Dios y el Estado. Dimensiones culturales del desarrollo político e institucional de América Latina. Nueva sociedad, 210, 78-94.

Picado, M. (1990). La iglesia costarricense entre el pueblo y el Estado: de 1949 a nuestros días. 2da edición. San José: Ediciones Guayacán.

Tschannen, O. (2004). La revaloración de la teoría de la secularización mediante la perspectiva comparada Europa Latina América Latina. En Jean Pierre Bastian 
(coord.), La modernidad religiosa. Europa latina y América Latina en perspectiva comparada. (pp.155-174). México D.F.: Fondo de Cultura Económica.
Vallarino Bracho, C. (2005). Laicidad y Estado moderno: definiciones y procesos. Cuestiones políticas. 34 , 157-173. 